\title{
Using feed-forward networks to infer the activity of feed-back neuronal networks
}

\author{
Xinxian Huang ${ }^{1 *}$, Farzan Nadim ${ }^{1,2}$, Amitabha Bose ${ }^{1,3}$ \\ From Nineteenth Annual Computational Neuroscience Meeting: CNS*2010 \\ San Antonio, TX, USA. 24-30 July 2010
}

Phase-locked activity of neurons is important for producing the proper activation sequence and coordination of neurons within Central Pattern Generating (CPG) networks. As it is often difficult to analyze the phase-locked activity of a large feedback network directly, it is critical to develop analytic methods that are lower-dimensional which involve different pieces of the full network. Here, we provide a technique to study smaller feed-forward networks and then combine the obtained information to understand the activity of feedback networks. As a case study, we study the phase of activity of two reciprocally inhibitory bursting neurons in the crustacean stomatogastric ganglion (STG) in which the firing time of one neuron has an effect on the period of the other, and vice versa. Each of these two processes defines a feedforward map and we combine them to determine the period and activity phase of the feedback network. We examine the conditions on the existence and stability of phase-locked solutions when the synapses display shortterm depression, a common form of synaptic plasticity. We find that the stability is lost in some circumstances in the feedback network with the existence of the synaptic depression.

Consider two neurons coupled via inhibitory synapses. Neuron A is modeled as oscillatory while neuron B as tonically active. The synapse from $\mathrm{A}$ to $\mathrm{B}$ is depressing but $B$ to $A$ is not. The cycle period of neuron $A$ depends on the firing time of $B$ relative to $A$, which

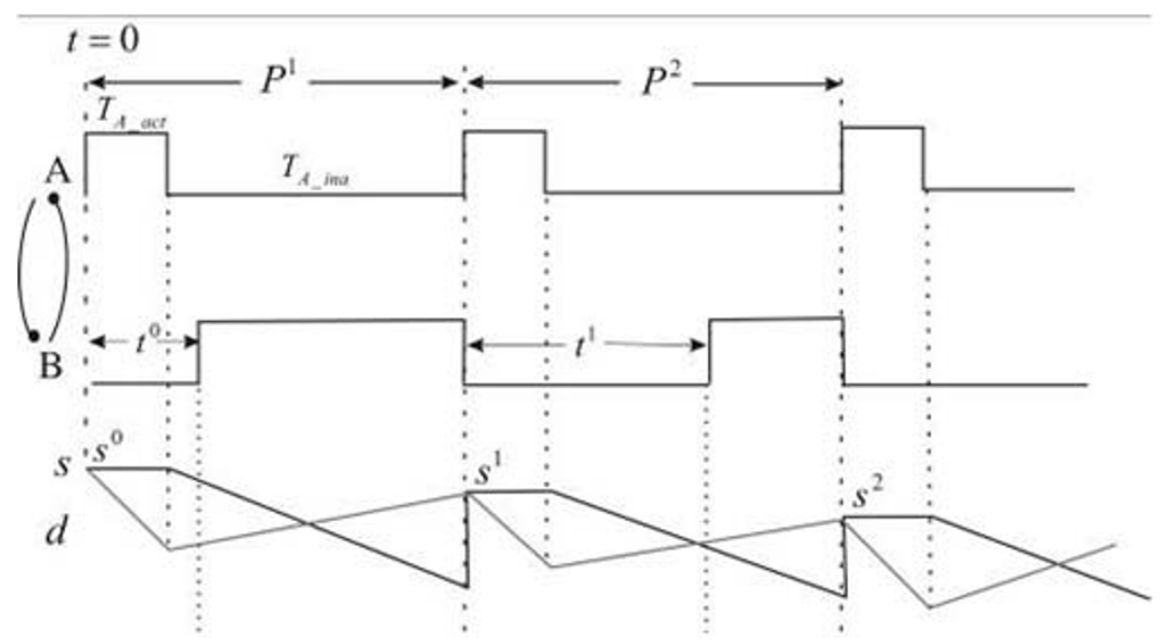

Figure 1 Reciprocally inhibitory network.

* Correspondence: xh28@njit.edu

1 Department of Mathematical Sciences, New Jersey Institute of Technology, Newark, NJ 07102, USA 


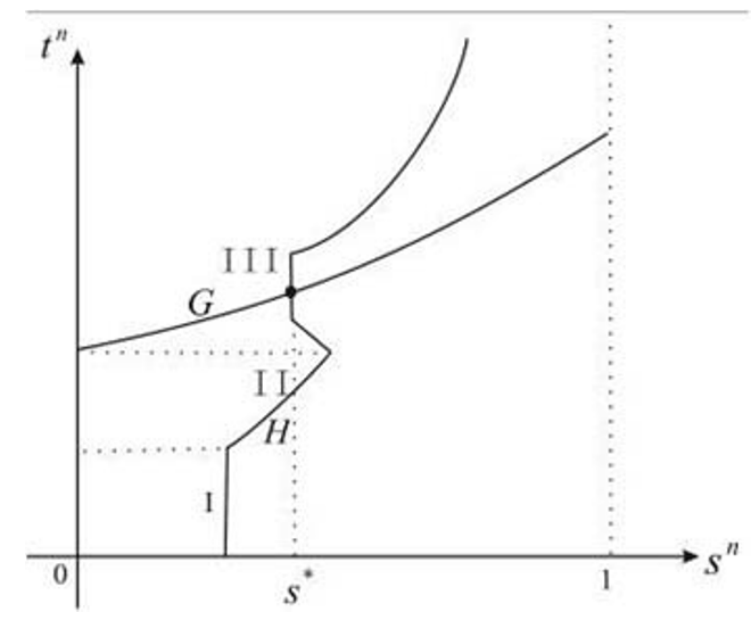

Figure 2 Intersection of feed-forward curves $H$ and $G$ correspond to a fixed point of the one-dimensional feedback map.

affects the recovery and strength of the synapse from A to $B$. This synapse, in turn, determines the firing time of $\mathrm{B}$ relative to $\mathrm{A}$. We derive a one-dimensional Poincare map $\Pi$, defined at the onset of A activity, that measures the strength $s^{n}$ of the A to B synapse (Fig. 1). The model is built as in [1]. Here $d$ measures the extent of depression of the A to B synapse, $t^{n}$ the firing time of $\mathrm{B}$ relative to $\mathrm{A}$ and $P^{n}$ the period of $\mathrm{A}$ in each cycle. A fixed point of this map determines the unique period and phase at which A and B lock. Its existence corresponds to the intersection point of the two feed-forward maps (Fig. 2), each of which is obtained from a different feedforward sub-network. One map, $H$, determines how $t^{n}$ affects $s^{n+1}$, while the other map, $G$, determines how $s^{n}$ affects $t^{n}$. The map $H$ is defined separately in three different regions. In Region I, neuron B fires when A is still active but its activity is not terminated by B; Region II is similar to I but the activity of A is terminated by B; in Region III, B fires when A is inactive. In biological networks Region I and II are small and the graphs of $H$ and $G$ typically intersect in Region III, as observed in the two mutually inhibitory neurons PD and LP in the STG. We find that the phase-locked solution is stable when it occurs in Regions I and III but the stability is lost in some circumstances in Region II.

\section{Acknowledgements}

Supported by NSF DMS-0615168 and NIH MH-60605.

\section{Author details}

'Department of Mathematical Sciences, New Jersey Institute of Technology, Newark, NJ 07102, USA . ²Department of Biological Sciences, Rutgers University, Newark, NJ 07102, USA. ${ }^{3}$ School of Physical Sciences, Jawaharlal Nehru University, New Delhi 110067 India.

Published: 20 July 2010

\section{Reference}

1. Bose A, Manor $Y$, Nadim F: The activity phase of postsynaptic neurons in a simplified rhythmic network. J Comput Neurosci 2004, 17:245-261.

doi:10.1186/1471-2202-11-S1-P48

Cite this article as: Huang et al:: Using feed-forward networks to infer the activity of feed-back neuronal networks. BMC Neuroscience 2010 11(Suppl 1):P48.
Submit your next manuscript to BioMed Central and take full advantage of:

- Convenient online submission

- Thorough peer review

- No space constraints or color figure charges

- Immediate publication on acceptance

- Inclusion in PubMed, CAS, Scopus and Google Scholar

- Research which is freely available for redistribution

Submit your manuscript at www.biomedcentral.com/submit 